Reprod. Nutr. Dévelop., 1982, 22 (1 A), 41-48.

\title{
Essais préliminaires sur la résistance aux hautes pressions du spermatozoïde de taureau, en vue d'une conservation à basse température, à l'état liquide
}

\author{
L. HENRIET, Françoise RENAUT, Annick VAN HOECKE
}

avec la collaboration technique de J.-H. MAISON et Thérèse BOGDAN

Université Catholique de Louvain.

Unité d'Embryologie et d'Anatomie animales.

Place Croix-du-Sud, 3 B, 1348 Louvain-la-Neuve.

Summary. Preliminary trials on the resistance of bull spermatozoa to high pressure in view of storing them under low pressure in the liquid state.

Diluted semen in Cassou ministraws was submitted to high pressure $(450,900$ or $1800 \mathrm{~kg} / \mathrm{cm}^{2}$ ) for 1,4 or $24 \mathrm{hrs}$ at $20^{\circ} \mathrm{C}$. After $1 \mathrm{hr}$ at $1800 \mathrm{~kg} / \mathrm{cm}^{2}$, all the spermatozoa were immotile. When the semen samples were submitted to $450 \mathrm{~kg} / \mathrm{cm}^{2}$ for 1 or $4 \mathrm{hrs}$, the motility percentages of both the control and treated groups were equal. The results obtained with $900 \mathrm{~kg} / \mathrm{cm}^{2}$ were intermediate.

\section{Introduction.}

L'influence de la pression sur les cellules et tissus n'est pas une préoccupation récente. De nombreux auteurs (Basset et Machebœuf, 1932, 1933 ; Basset et al., 1935a, $b$; Lauffer et Dow, 1941 ; Haubrich, 1937) ont observé ses effets sur les microorganismes, Dow et Matthews (1939) sur les érythrocytes et Marsland et Rugh (1940) sur le sperme de Rana pipiens. En 1973, Moßner s'est intéressé au sperme de taureau, tandis que Carter et al., la même année, publiaient leurs observations sur le rein et sur le spermatozoïde.

Nos premiers essais (1960, non publiés) avaient pour but la recherche d'un moyen de conservation du sperme, simple, peu coûteux et plus sûr que la congélation en présence de cryoprotecteurs. En effet, la présence de cristaux de glace au sein des cellules exerce une action destructrice et les cryoprotecteurs abaissent le pouvoir fécondant.

Ces deux inconvénients peuvent être éliminés par la conservation sous pression. Le diagramme des températures et pressions montre qu'il faut appliquer environ $100 \mathrm{~kg}$ de pression par degré en dessous de zéro pour prévenir la cristallisation. 
Les travaux publiés précédemment visaient à établir les conditions optimales de température et de pression pour obtenir une conservation satisfaisante du sperme de taureau (Henriet et al., 1977, 1979). Cependant, comme l'application de la pression doit précéder l'abaissement de la température pour que soit respectée l'intégrité cellulaire et puisque le temps nécessaire pour obtenir des pressions de l'ordre de $2000 \mathrm{~kg} / \mathrm{cm}^{2}$ est d'environ $5 \mathrm{~h}$, nous avons jugé indispensable de tester la résistance des cellules à la pression. Nous rapportons donc dans cet article les essais visant à déterminer la zone de pressions tolérées par le spermatozoïde sans que soit affectés sa motilité et ses caractères structuraux.

\section{Matériel et méthodes.}

1. Le sperme et le dilueur. - Le sperme, recueilli selon la méthode classique, est immédiatement placé dans une étuve à $32^{\circ} \mathrm{C}$.

Une première qualification est établie d'emblée en évaluant la motilité d'ensemble. Les échantillons n'atteignant pas une motilité de 70 p. 100 sont éliminés. Le sperme frais est dilué sans délai à raison d'une partie pour deux ou pour trois, avec un dilueur au jaune d'œuf de composition classique $\left({ }^{1}\right)$, porté à $32{ }^{\circ} \mathrm{C}$. Cette température, à laquelle s'opère la dilution au Centre qui fournit la semence, a été maintenue pour des raisons pratiques. Après cette dilution, le sperme est protégé contre le choc thermique et il peut se refroidir à la température du laboratoire.

2. Les pressions. $-5 \mathrm{~cm}^{3}$ de sperme sont répartis dans des « paillettes de Cassou " $(0,25 \mathrm{cc})$, qui sont bouchées et transférées dans un bain à la température de $20^{\circ} \mathrm{C}$. Après quelques minutes, les paillettes sont enfermées dans la chambre et soumises à la pression de 450,900 ou $1800 \mathrm{~kg} / \mathrm{cm}^{2}$ pendant 1,4 ou $24 \mathrm{~h}$. L'appareillage utilisé pour atteindre ces pressions a déjà fait l'objet d'une description (Henriet et al., 1977, 1979) : 2 compresseurs, de principe et de rayon d'action différents, sont utilisés successivement lorsque la pression à atteindre dépasse $1000 \mathrm{~kg} / \mathrm{cm}^{2}$. Le liquide médiateur de pression est une huile minérale inerte qui remplit tout le système.

La compression et la décompression sont effectuées progressivement en 5 à 15 min., de manière à ne pas causer de lésion au niveau du sperme (Moßner, 1973).

Les tests de $1 \mathrm{~h}$ sont effectués à $20^{\circ} \mathrm{C}$, celui de $24 \mathrm{~h}$, à $4{ }^{\circ} \mathrm{C}$. Les paillettes témoins sont laissées à des températures identiques, sans être soumises à une pression supérieure à la pression atmosphérique.

3. Les paramètres testés. - Trois méthodes ont été utilisées pour comparer la qualité des échantillons et des témoins avant et après expérience ; ce sont la

(1) Le dilueur est composé de : Tris, hydroxyméthylaminométhane : $24,22 \mathrm{~g}$; Glycocolle : $9,39 \mathrm{~g}$; acide citrique : $13,54 \mathrm{~g}$; glucose et fructose : aa $7,5 \mathrm{~g}$; pénicilline et streptomycine : aa $2 \mathrm{~g}$; eau bidistillée ad $1000 \mathrm{~g}$; additionné de jaune d'œuf ad 10 p. 100. 
mobilité, le temps de décoloration du bleu de méthylène et l'aspect morphologique.

a) La mobilité du sperme. Comme il est dit plus haut, la mobilité donne une réponse immédiate pour autant que les échantillons soient portés à température comparable. Toutefois cette mobilité n'est ni une valeur absolue, ni une valeur constante, elle est au contraire une variable échelonnée selon d'innombrables degrés. Cette variable a été évaluée par détermination de la mobilité d'ensemble. Deux opérateurs indépendants font l'examen en goutte pendante $\left(^{2}\right)$ à $37^{\circ} \mathrm{C}$. Pour ce faire, le sperme est réchauffé, comme le préconisent De Vuyst et al. (1959) et, beaucoup plus tard, Proudfoot (1972). Cette opération rapide s'est révélée d'une précision suffisante dans le cas présent ; en effet, pour lever les doutes à ce sujet, nous avons, à titre de comparaison, évalué le pourcentage de spermatozoïdes mobiles pour certains échantillons en cellules de Thoma, suivant la méthode spécifique décrite par De Vuyst et al. (1959) pour les tests de fécondité et reprise plus tard par Makler (1978).

b) La décoloration du bleu de méthylène. On effectue rapidement un test de décoloration du bleu de méthylène à $37^{\circ} \mathrm{C}$ en suivant la technique de Milanov et Smirnov-Ougrioumov (De Vuyst et al., 1959). Une réaction témoin, en présence de dilueur seul nous a montré que celui-ci, en raison de la présence de lactate et de pyruvate, opérait la décoloration totale du bleu de méthylène en $50 \mathrm{~min}$.

c) Les frottis de sperme. Des frottis de sperme témoin et soumis à la pression ont été réalisés lors de chaque expérience. Ils ont été colorés ultérieurement au Wasserblau et au Rose Bengale (De Vuyst et Henriet, 1965) de manière à rendre plus discernables des lésions perceptibles au microscope optique. Cette coloration met bien l'acrosome et les membranes en évidence et notamment leurs altérations qui sont un indice soit de maturation, soit de mortalité, et en tous cas de vieillissement (Henriet et Horvat, 1976).

Remarque : On constatera que nous insistons sur le choix des températures et sur la promptitude d'exécution. Si on se réfère à l'équation générale de la fructolyse (tabl. 1), on saisit l'importance de ces deux conditions, que ce soit pour des contrôles comparatifs ou pour l'efficacité de la conservation. C'est pourquoi les manipulations sont réalisées très rapidement et à température constante.

$$
\begin{gathered}
\text { TABLEAU } 1 \\
\text { Equation de la fructolyse } \\
\text { Facteurs endogènes }\left\{\begin{array}{c}
\text { milieu : } \mathrm{H}_{2} \mathrm{O} \\
\text { substances énergétiques } \underset{\mathrm{m}}{\longrightarrow} \text { métabolites }+ \text { énergie } \\
+ \text { enzymes }
\end{array}\right. \\
\text { Facteurs exogènes }\left\{\begin{array}{c}
\mathrm{pH} \text { initial } \underset{\text { variation de } \mathrm{pH}}{+} \\
( \pm \text { bactéries })
\end{array}\right.
\end{gathered}
$$

(2) II s'agit d'un examen inversé ; une goutte de sperme est suspendue à la face inférieure d'un couvre-objet, reposant sur un porte-objet évidé. On évite ainsi les déformations optiques dues à la sphéricité de la goutte, ou encore, le frein exercé par le poids d'un couvre-objet qui rétablit la planéité de la préparation. 


\section{Résultats.}

1. Lésions et vieillissement. - Les spermatozoïdes, aux pressions exercées, restent morphołogiquement intacts, si l'on en juge d'après les frottis examinés au microscope optique. On ne constate pas d'éclatement, d'écrasement ni de fracture. De plus, les acrosomes et les membranes ne sont pas plus altérés, dans la proportion de survie observée sous chacune de ces pressions, ce qui est l'indice d'un frein au vieillissement.

2. La mobilité et la survie. - Les pourcentages de mortalité évalués avant et après la compression, sont comparés dans les figures 1 et 2 .

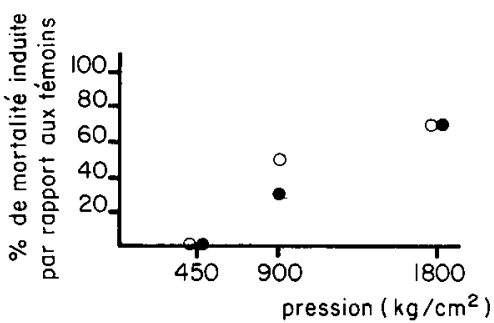

FIG. 1. - Résistance à la pression pendant $1 \mathrm{~h}$ (Evaluation par la mobilité).

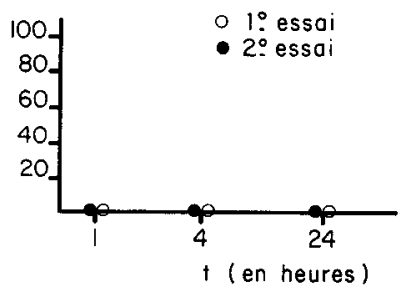

FIG. 2. - Résistance à la pression de $450 \mathrm{~kg} / \mathrm{cm}^{2}$ au cours du temps (Evaluation par la mobilité).

Leur examen permet de conclure qu'une pression de $1800 \mathrm{~kg} / \mathrm{cm}^{2}$, exercée pendant $1 \mathrm{~h}$, tue les spermatozoïdes. En effet, la durée de décoloration du bleu de méthylène atteint $50 \mathrm{~min}$. ; elle est équivalente à celle mesurée en présence du dilueur seul (Henriet, 1958) (voir tabl. 2).

\section{TABLEAU 2}

Temps de réduction du bleu de méthylène pour le sperme soumis pendant $1 \mathrm{~h}$ à une pression supérieure à la pression atmosphérique

\begin{tabular}{|c|c|c|c|}
\hline & \multicolumn{3}{|c|}{ Pression en $\mathrm{kg} / \mathrm{cm}^{2}$ de l'échantillon traité } \\
\hline & 450 & 900 & 1800 \\
\hline 1er essai : $\begin{array}{c}\text { Témoin } \ldots \ldots \ldots \ldots \ldots \ldots \ldots \ldots \ldots \\
\text { Traité } \ldots \ldots \ldots \ldots \ldots \ldots \ldots \ldots\end{array}$ & $\begin{array}{l}10 \mathrm{~min} . \\
10 \mathrm{~min} .\end{array}$ & $\begin{array}{l}15 \mathrm{~min} . \\
60 \mathrm{~min} .\end{array}$ & $\begin{array}{l}25 \mathrm{~min} . \\
40 \mathrm{~min} .\end{array}$ \\
\hline 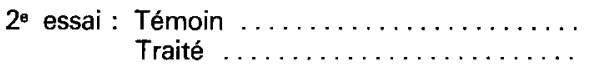 & $\begin{array}{l}25 \mathrm{~min} . \\
25 \mathrm{~min} .\end{array}$ & $\begin{array}{l}15 \mathrm{~min} . \\
15 \mathrm{~min} .\end{array}$ & $\begin{array}{l}20 \mathrm{~min} . \\
60 \mathrm{~min} .\end{array}$ \\
\hline
\end{tabular}

C'est donc très vraisemblablement la mort de la cellule qui est responsable de son immobilité et non une lésion qui serait limitée aux éléments moteurs seuls. Effectivement, une fracture des éléments contractiles n'influencerait pas automatiquement un ralentissement du métabolisme (Henriet et al., 1979). 
Par contre, une pression de $450 \mathrm{~kg} / \mathrm{cm}^{2}$, exercée pendant $1 \mathrm{~h}, \mathrm{n}^{\prime}$ affecte ni la mobilité, ni le temps de décoloration du bleu de méthylène.

Quant à la pression de $900 \mathrm{~kg} / \mathrm{cm}^{2}$, elle a des effets intermédiaires et induit, en moyenne, une chute de la mobilité de 30 p. 100, avec, selon les cas, un allongement du temps de décoloration du bleu de méthylène.

Si I'on considère l'effet de l'allongement du temps de compression pour une pression optimale de $450 \mathrm{~kg} / \mathrm{cm}^{2}$ (fig. 2), on constate que le sperme de taureau résiste bien pendant 1 à $4 \mathrm{~h}$. La résistance est encore très acceptable, si la durée de compression est portée à $24 \mathrm{~h}$.

Dans ces essais, la durée de décoloration du bleu de méthylène est identique pour les échantillons témoins et les échantillons traités (tabl. 3).

\section{TABLEAU 3}

Temps de réduction du bleu de méthy/ène du sperme soumis à $450 \mathrm{~kg} / \mathrm{cm}^{2}$ pendant $1 \mathrm{~h}, 4 \mathrm{~h}$ et $24 \mathrm{~h}$

\begin{tabular}{|c|c|c|c|c|}
\hline & & \multicolumn{3}{|c|}{ Durée de la mise sous presssion } \\
\hline & & $1 \mathrm{~h}$ & $4 \mathrm{~h}$ & $24 \mathrm{~h}$ \\
\hline $1^{\text {er }}$ essai & $\begin{array}{l}\text { : Témoin } \\
\text { Traité . }\end{array}$ & $\begin{array}{l}10 \mathrm{~min} . \\
10 \mathrm{~min} .\end{array}$ & $\begin{array}{l}10 \mathrm{~min} . \\
15 \mathrm{~min} .\end{array}$ & $\begin{array}{l}13 \mathrm{~min} . \\
12 \mathrm{~min} .\end{array}$ \\
\hline $2^{e}$ essai : & $\begin{array}{l}\text { Témoin } \\
\text { Traité . . }\end{array}$ & $\begin{array}{l}25 \mathrm{~min} . \\
25 \mathrm{~min} .\end{array}$ & $\begin{array}{l}10 \mathrm{~min} . \\
10 \mathrm{~min} .\end{array}$ & $\begin{array}{l}10 \mathrm{~min} . \\
15 \mathrm{~min} .\end{array}$ \\
\hline
\end{tabular}

\section{Discussion.}

Cette étude nous a permis de déterminer la gamme de pressions à laquelle résiste le spermatozoïde de taureau, pendant 1 à $24 \mathrm{~h}$, à la température de $20^{\circ} \mathrm{C}$ ou de $4^{\circ} \mathrm{C}$.

En 1973, Moßner a effectué un essai analogue sur du sperme de taureau, à 50 ou à $100 \mathrm{Atm}$, pendant $5 \mathrm{~min}$., sans se préoccuper de la température. II a constaté une diminution de motilité et une augmentation du pourcentage d'acrosomes endommagés. II s'est intéressé à l'effet de la fertilité et, conformément aux conclusions de Saacke et al. (1968), et de Saacke et Marshall (1968), qui attribuent à l'intégrité de l'acrosome, un grand rôle dans la valeur fertilisante du sperme, il conclut à une détérioration de la fécondité par la pression. Ses conclusions ne concordent pas avec les nôtres et, à l'analyse, il se révèle qu'il provoque une action destructive à des pressions bien inférieures à celles que nous avons exercées. Dans la précédente publication (Henriet et al., 1979), nous avons rendu compte de l'effet de pressions de $1600 \mathrm{~kg} / \mathrm{cm}^{2}$ et, si les spermatozoïdes étaient morts, c'était à la suite de lésions très discrètes, puisque nous n'avons pas décelé de dommages au microscope optique. Il en est de même dans ce second essai. 
Le contrôle des paramètres s'est déroulé différemment de celui de Moßner, puisque celui-ci réalise seulement après $24 \mathrm{~h}$ ce que nous avons pratiqué directement après la décompression.

A l'échelle du spermatozoïde, $24 \mathrm{~h}$ représentent un long délai. Or cette cellule est sensible aux modifications de milieu, qu'elle traduit par des altérations notamment au niveau de l'acrosome. Dans de telles conditions, les lésions de l'acrosome ne sembleraient pas devoir être mises en corrélation avec le seul effet de la pression.

Si on se remémore l'équation de la fructolyse (tabl. 1) on comprend mieux les précautions à prendre avant la mise sous pression, le choix des paramètres, leur signification.

Saacke et al. (1968) estiment que le pouvoir fécondant est lié à l'intégrité de l'acrosome. Si nous ne trouvons pas d'altérations de l'acrosome dans des conditions expérimentales plus drastiques, c'est qu'il a été tenu compte de l'équation fondamentale, tout au long des opérations. Le sperme est dilué pour permettre son refroidissement et son ravitaillement en énergie. Le contrôle de motilité et de décoloration du bleu de méthylène est effectué à chaud, pour obtenir l'efficacité optimale des enzymes.

La décoloration au bleu de méthylène nous a été suggérée par Sollozy (communication verbale) qui, plutôt que la mort des spermatozoïdes, imaginait une fracture des éléments contractiles. Dougherty et al. (1975) avaient par ailleurs montré que l'immobilité ne signifiait pas mort, tout comme le blocage du mouvement par l'acide lactique ne tue pas le spermatozoïde.

Le résultat est malgré tout négatif et, lorsque les spermatozoïdes retirés de la chambre sont immobiles, ils ne décolorent pas le bleu de méthylène plus rapidement que le dilueur seul : ils sont donc morts.

De ce qui précède, on comprendra aisément pourquoi cet essai au-dessus de zéro degré, s'est limité à $24 \mathrm{~h}$. On savait d'avance que du sperme non refroidi s'altérerait, qu'il soit ou non sous pression. La proposition n'était pas, dans ce cas, d'atteindre une longue durée, mais uniquement de connaître les limites supportables de pression.

\section{Conclusions.}

Nous ne pouvons rien affirmer quant au pouvoir fécondant du sperme soumis à une pression inférieure à $1000 \mathrm{~kg} / \mathrm{cm}^{2}$, à température ordinaire. Nous pouvons seulement prévoir qu'il sera peu ou pas affecté, tout au moins jusqu'à $-4,5^{\circ} \mathrm{C}$, sous $450 \mathrm{~kg} / \mathrm{cm}^{2}$ de pression, puisque dans ces conditions les échantillons sont restitués dans un état identique à celui des témoins. La conservation se situerait ainsi à moyen terme. Bien que nous sachions au départ que la zone de pressions utilisables était limitée, nous espérions pouvoir descendre cependant à des températures qui, a priori, nous semblaient plus efficaces. 
Remerciements. - Ce travail a été accompli dans le cadre de notre collaboration avec le Gouvernement Provincial du Brabant que nous remercions ici.

Nous remercions également M. le Dr G. Jaumotte, Directeur du Centre d'Insémination de Wavre, qui nous a fourni les prélèvements nécessaires à ces expérimentations, ainsi que M. P. Estermans pour les multiples conseils techniques qu'il nous a donnés dans le domaine des hautes pressions.

\section{Références}

BASSET J., MACHEBOEUF M., 1932. Etude sur les effets biologiques des ultrapressions ; résistance des bactéries, des diastases et des toxines aux pressions très élevées. $C$. $R$. Acad. Sci., $195,1431$.

BASSET J., MACHEBOEUF M., 1933. Etudes sur les effets biologiques des ultrapressions. Etude sur l'immunité : influence des pressions très élevées sur certains antigènes et anticorps. C. R. Acad. Sci., 196, 67.

BASSET J., MACHEBOEUF M., 1933. Note résumant les résultats obtenus dans l'étude de I'influence de très hautes pressions sur les microorganismes et sur les ferments. Ergeb. $d$. Enzymfor. Leipzig, 1, 304.

BASSET J., WOLLMAN E., MACHEBCEUF M., 1935a. Etudes sur les effets biologiques des ultrapressions. Action des pressions très élevées sur les bactériophages des spores et sur les autolysines. C. R. Acad. Sci., 200, 1072.

BASSET J., NICOLAU S., MACHEBOEUF M., 1935b. L'action de l'ultrapression sur l'activité pathogène de quelques virus. C. R. Acad. Sci, 200, 1882.

BRIDGMAN P. W., 1912. High pressures and five kinds of ice. J. Franklin Inst., vol, 177, 315-332.

CARTER J. E., RISCH S. J., GRAHAM E. F., LILLEHEI R. C., 1973. The effect of pressure and cooling rate on spermatozoa, kidney tissue and the whole kidney. Cryobiology, 10, 263270.

DE VUYST A., MOREELS A., HENRIET L., ARNOULD R., VERVACK W., VANBELLE M., 1959. La valeur d'un test combiné bleu de méthylène-spermatozoïdes vivants pour la détermination de la valeur du sperme. Ann. Méd. vét., 1, 7-26.

DE VUYST A., HENRIET L., 1960. Le blocage du métabolisme du spermatozoïde par voie chimique. 25e Anniv. Fond. Ist. Ital. Lazarro Spallanzani, 1-5.

DE VUYST A., HENRIET L., 1965. Observations sur la coiffe céphalique du spermatozoïde de taureau. Zootechnia, 14, 7 pp.

DOUGHERTY K. A., EMILSON L. B. V., COCKETT A. T. K., URRY R. L., 1975. A comparison of subjective measurements of human sperm motility and viability with two live-dead staining techniques. Fert. Steril., 26, 700-703.

DOW R. B., MATTHEWS J. E., 1939. The desintegration of erythrocytes and denaturation of hemoglobin by high pressure. Phil. Mag., 27, 637.

EISENBERG D., KAUZMANN, 1969. The structure and properties of water. Oxford at the Clarendon Press, $93 \mathrm{pp}$.

HAUBRICH R., 1937. The resistance of the erythrocytes to pressure. Arch. ges. Physiol., 239, 304. HENRIET L., 1958. Biologie clinique des liquides spermatiques. Conf. Soc. Biol. clin. Liège.

HENRIET L., HORVAT F., 1976. Glycoprotein layer on bull spermatozoa investigated in vivo and in vitro. VIII' Congr. int. Reprod. anim. Insém. artif. Cracovie.

HENRIET L., ESTERMANS P., MOTTOUL B., MAISON J.-H., 1977. Manipulation of egg in vitro ; attempt at long term storage of oocytes at low temperatures without freezing, 450-472. In SREENAN J. M., Control of reproduction in the cow. CEE Luxembourg, Martinus Nijhoff, La Haye.

HENRIET L., ESTERMANS P., CUCHE B., MAISON J.-H., 1979. Essai de conservation prolongée de gamètes à basse température, sans congélation. Ann. Biol. anim. Bioch. Biophys., 19, 367-373. 
LAUFFER M. A., DOW R. B., 1941. The denaturation of tobacco mosaic virus at high pressure. J. biol. Chem., 140, 508-518.

MAKLER A., 1978. A new chamber for rapid sperm count and motility estimation. Fert. Steril, 30, 313-318.

MARSLAND D. A., RUGH R., 1940. Resistance of sperm of Rana pipiens to hydrostatic compression ; effect upon embryonic development. Proc. Soc. exp. Biol. Med., 43, 141-143.

MOSNER H. U., 1973. Uber die Druckresistenz von Samenzellen. Inaugur. Diss. Aus dem Institut für Haustierbesamung und Andrologie der Tierärztlichen Hochschule Hannover, $74 \mathrm{pp}$.

PROUDFOOT F. G., 1972. Effects of high pressure gases on the motility and fertilizing capacity of avian spermatozoa stored in vitro. J. Reprod. Fert., 31, 367-371.

SAACKE R. G., MARSHALL C. E., 1968. Observations on the acrosomal cap of fixed and unfixed bovine spermatozoa. J. Reprod. Fert., 16, 511-514.

SAACKE R. G., AMANN R. P., MARSHALL C. E., 1968. Acrosomal cap abnormalities of sperm from subfertile bulls. J. anim. Sci., 27, 1391-1400. 